\title{
Potency Assay
}

National Cancer Institute

\section{Source}

National Cancer Institute. Potency Assay. NCI Thesaurus. Code C134270.

The determination of the strength of a material, as measured by the ability to produce a specific response. The potency of a pharmacologic substance is measured by the amount needed to produce a certain effect. 